\title{
Review Article: Postmortem Liver Pathology Findings in Patients With COVID-19: A Systematic Review
}

\author{
Mehdi Forouzesh $^{1}$ D, Behnam Behnoush ${ }^{2}$, Anahita Sadeghi ${ }^{3}$, Hengameh Shahnavaz $^{4}$, Abdolrazagh Barzegar $^{1}$, Elham Bazmi $^{* *}$ id
}

1. Legal Medicine Research Center, Legal Medicine Organization, Tehran, Iran.

2. Department of Forensic Medicine, Faculty of Medicine, Tehran, Iran.

3. Digestive Disease Research Center, Digestive Diseases Research Institute, Tehran University of Medical Science, Tehran, Iran.

4. Department of Pathology, School of Medicine, Tehran University of Medical Sciences, Tehran, Iran.

\begin{tabular}{|c|c|}
\hline $\begin{array}{l}\text { Use evour device to scan } \\
\text { and read the article online }\end{array}$ & ditation Forouzesh M, Behnoush B, Sadeghi A, Shahnavaz H, Barzegar A, Bazmi E. Postmortem Liver Pathology Findings \\
\hline 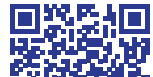 & $\begin{array}{l}\text { in Patients With COVID-19: A Systematic Review. International Journal of Medical Toxicology and Forensic Medicine. 2021; } \\
\text { 11(4):35042. https://doi.org/10.32598/ijmtfm.v11i4.35042 }\end{array}$ \\
\hline ( & d.I https://doi.org/10.32598/ijmttm.v11i4.35042 \\
\hline
\end{tabular}

(c) (i) (5)

Article info:

Received: 29 May 2021

First Revision: 21 Jun 2021

Accepted: 10 Jul 2021

Published: 21 Dec 2021

\section{Keywords:}

Postmortem, Findings, Liver, Pathology, Coronavirus Disease 2019 (COVID-19)

\section{ABSTRACT}

Background: The Coronavirus Disease 2019 (COVID-19) pandemic promptly became a significant public health challenge with extra-pulmonary manifestations, including liver damage. Postmortem examination is crucial for gaining a better understanding of these manifestations and improving patient management. This study summarized the current knowledge of the postmortem liver pathology of patients with COVID-19.

Methods: This review was conducted on studies evaluating the postmortem macroscopic and microscopic findings of the liver in patients with COVID-19. Accordingly, we searched 4 electronic databases (PubMed, Scopus, Google Scholar, \& Web of Science) until June 2021. From the 317 screened articles, 16 articles examining a total of 332 patients who had died due to COVID-19 were selected.

Results: The significant findings of the liver were moderate macro and microvesicular steatosis with mild sinusoidal dilation, active lobular and portal vein thrombosis, mildly-increased lymphocyte filtration in sinusoidal space, and multifocal hepatic necrosis. Additionally, the most common comorbidities were hypertension and other metabolic diseases. In conclusion, liver damage due to COVID-19 infection has various manifestations in patients who have expired due to COVID-19.

Conclusion: Therefore, monitoring liver function during the course and treatment of this disease is necessary for better patient management and to decrease the COVID-19-induced mortality rate COVID.

\section{* Corresponding Author: \\ Elham Bazmi, PharmD, PhD.}

Address: Legal Medicine Research Center, Legal Medicine Organization, Tehran, Iran

Tel: +98 (98) 77532534

E-mail:drelbazmi@yahoo.com 


\section{Introduction}

he recent Coronavirus Disease 2019 (CO$\mathbf{T}$ VID-19) has rapidly spread worldwide. COVID-19 was declared a pandemic by the World Health Organization (WHO) on March 11, 2020. The outbreak of COVID-19 has become a significant public health challenge over the past months $[1,2]$.

The COVID-19 has not been previously found in humans; up to now, no specific effective antiviral therapies have been identified for it. Besides, the virus infection diagnosis is difficult and crucial in most cases [3, 4].

Researchers worldwide are progressing in controlling and treating this infection; however, little is known about pathological postmortem findings from patients who have expired due to COVID-19 in their extra-respiratory organs.

Autopsy findings are essential for understanding how COVID-19 infection affects the human body as applied to the clinic for improved diagnosis and treatment strategies [5].

Respiratory failure seems to be the leading cause of death in patients with COVID-19. However, in approximately $26 \%$ of the patients, the infection can lead to various severe conditions with multiple organ failure that could cause death; if these failures are diagnosed and managed adequately on time, death is preventable, even in these instances [6-9].

Liver involvement was predictable in COVID-19 infection, just like the Middle East Respiratory Syndrome (MERS) and Severe Acute Respiratory Syndrome (SARS) [10]. The mechanism of hepatic injury may differ in different patients and is multifactorial, consisting of the following:

1. The direct viral attack and hepatic necrosis are consistent with the pattern of acute liver injury and cause an elevation of serum transaminases, such as alkaline phosphatase and Gamma-Glutamyl Transferase (GGT), in some patients [11].

2. Drugs used in treating COVID-19, such as azithromycin and hydroxychloroquine, can cause various degrees of hepatotoxicity [12].

3. Secondary injuries in patients with underlying liver diseases include chronic viral hepatitis, nonalcoholic fatty liver, and Cirrhosis [13].
4. A hyper-inflammatory reaction to COVID-19 can exacerbate the production of pro-inflammatory cytokines $[10,14]$.

5. Expression of ACE2 in the liver and bile duct epithelial cells in patients with COVID-19 can cause liver injury. The level of ACE2 expression in cholangiocytes resembles type 2 alveolar cells of the lungs, indicating that the liver can be a potential target for COVID-19 [15].

The present systematic review summarizes the current knowledge about macroscopic and microscopic findings of the liver tissue in patients with COVID-19 in postmortem examinations.

\section{Materials and Methods}

This study was performed based on the Preferred Reporting Items for Systematic Reviews (PRISMA) standards [16]. A search was conducted in four online databases (PubMed, Scopus, Google Scholar, \& Web of Science) for published June 1, 2021. The search keywords were employed according to the following search string: "Coronavirus" OR "COVID-19" OR "SARSCoV-2" AND "chronic liver injury" OR "acute liver injury" OR "cirrhosis" AND "postmortem" OR "autopsy". The electronic search strategy for all the four databases is detailed in Supplementary Table 1.

The data collection process, including literature selection and data selection, was performed independently by three authors (BB, EB, \& MF), who searched for titles or abstracts related to liver findings for death due to COVID-19. Disagreements between the authors about the eligibility of any of the articles were resolved through consensus or by adding a fourth researcher (AS) to make the final decision.

The extracted studies were screened based on the predefined article eligibility criteria, including a) Being published in English only; b) Reporting on macroscopic or microscopic findings for the liver in COVID-19-related death; c) Including demographic data, comorbidities, and autopsy details of the cadavers. The studies were excluded if they were: a) published in languages other than English; b) in vitro or an animal research; c) a review article, commentary, correspondence, editorial, and case report with a sample size of less than five; d) missing relevant information; e) involved pregnant women and children. The duplicate studies were excluded.

The quality of the included studies was evaluated using the Newcastle-Ottawa Quality Assessment Scale (NOS) 
tool that was applied for quality assessment of case series and cohort studies in a systematic review and metaanalyses [17]. Two authors independently assessed the included studies to reduce the risk of bias. The risk of bias of this systematic review regards data about pre-existing or concomitant pathology resulting from micro or each article reported macroscopic findings and comorbidity.

\section{Results}

\section{Literature search}

Our search in four scientific databases yielded 220 unique articles in this systematic review study. After screening the relevant titles and abstracts, 121 full-text articles were assessed for eligibility. These studies were carefully evaluated according to the inclusion criteria and the main objectives of the review. Finally, 16 articles were included in the study [18-33]. Figure 1 presents the search strategy as a PRISMA flowchart showing the selection process.

\section{Quality analysis}

The quality analysis of the included articles was performed based on the following criteria:

1) Reporting on more than 10 cases; 2) reporting macroscopic evaluation results for the liver for each case; 3 ) reporting microscopic evaluation results for each case. Articles with all three criteria were considered to be of high quality. Articles without macroscopic or microscopic data and higher than 10 cases were supposed to be of moderate quality. Articles without macroscopic or microscopic data and less than 10 cases were considered low quality. Of the 16 papers included in the study, $6(37.5 \%)$ studies were determined to be high quality and $8(50 \%)$ moderate quality. Finally, two articles $(12.5 \%)$ were of low quality. Supplementary Table 2 demonstrates the quality analysis results of the included studies.

\section{Characteristics of the studies}

Of the 16 included studies, 14 were case series, and two had a prospective design. Two of the 16 selected studies reported multicenter data $[21,28]$. while the remaining 14 represented single-center data. The included articles described a total of 332 cadavers, with a mean age of 67.6 years. Among them, 220(66\%) were males, and $112(34 \%)$ were females. The postmortem examination was conducted as a complete autopsy in $44 \%$ of the studies and a minimally invasive autopsy or both in the remaining cases. All studies performed a histological evaluation of the liver tissue. Table 1 lists the characteristics of the studies enrolled in this systematic review.

\section{Macroscopic and microscopic findings from the liver specimens}

The gross examination and liver histopathology findings were described for 131 and 311 patients, respectively. Table 2 summarizes the main conclusions of the reviewed articles on COVID-19-related deaths.

\section{Macroscopic findings from the liver specimens}

Schmit et al. described their macroscopic examination of the liver on 14 cadavers with COVID-19 infection; $57 \%$ of the livers examined by them had a pale, yellowish appearance, and $42 \%$ had a nutmeg appearance. In $14 \%$ of the livers, indurated consistency and slight irregularity were seen, and an indurated whitish nodule was diagnosed in one of them. Only one liver represented no macroscopic particularities. The median weight of the livers was 1987 (1280-3220) grams [27].

Sonzogni et al. published a case series of 48 patients who had died from COVID-19 in two leading hospitals in northern Italy. The authors reported minimal inflammation features during all the liver specimens [28].

Wichmann et al. performed a complete autopsy on 12 COVID-19 positive deaths. Hepatomegaly was observed in one of them and shock liver in three patients. Furthermore, two dead patients had chronic congestion and fatty liver changes [29].

In the macroscopic evaluation of 40 dying patients with COVID-19, Lagana et al. showed fibrosis and abscess in two liver samples with varying degrees of steatosis. At the same time, ischemia and congestion were diagnosed in the remaining specimens [32].

Fassan et al. detected a zone 3 sinusoidal ectasia with significant red cells congestion in 21 cases and signs of centrilobular parenchymal atrophy in 5 from 25 cadavers [33].

\section{Microscopic findings from the liver specimens}

One of the most common microscopic findings in the liver tissue in the included articles was micro or macrovesicular steatosis, i.e., observed in $178(57 \%)$ of the dead COVID-19 patients [18, 19, 21-25, 27, 31-33].

In the study by Sadegh Beigee et al., postmortem lung and liver samples were obtained from 31 deceased patients with COVID-19. The liver histopathological 

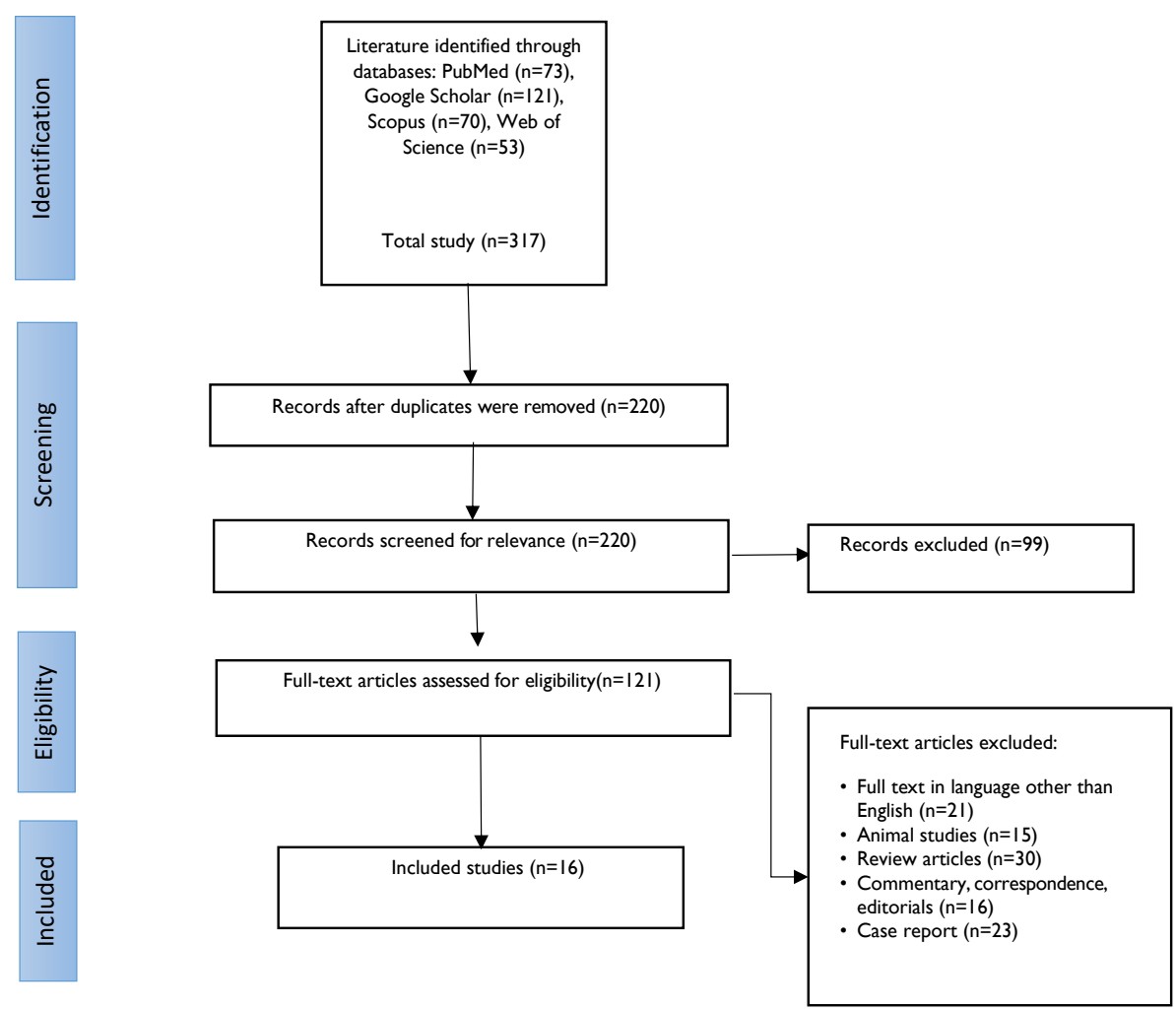

Figure 1. PRISMA flowchart showing study selection process for systematic review

International Journal of International Journal of
Medical Toxicology \& Forensic Medicine

examination indicated the fatty change in $59 \%$ of the specimens with macro or microvesicular patterns of involvement. Besides, mild to severe portal lymphoplasmacytic inflammation was reported in $59 \%$ of the cases with interface hepatitis in the other samples and passive congestion in $70 \%$ of them. Hepatocellular injury and lobular inflammation were observed in some liver specimens. Extensive parenchymal brown pigmentation and multifocal parenchymal necrosis with suspicious intracytoplasmic inclusions were detected in $7 \%$ and $4 \%$ of the liver tissues, respectively [18].

In the study by Beigmohammadi et al., mild to severe forms of congestion and sinusoidal dilatation were among the main findings in the microscopic examination of the liver in the patients who had died with COVID-19 infection. Furthermore, the researchers reported focal and scattered bile plugs, focal confluent necrosis, and hepatocyte dropout in part of the cadavers [19].

Bradley et al. performed minimally invasive autopsies on 14 patients with SARS-CoV-2 infection, and their histopathological data of the liver demonstrated sinusoidal congestion and fibrin thrombosis [20].
Buja et al. published a case series of 23 patients with COVID-19 from 5 centers in the USA. Lymphoplasmacytic triaditis with portal fibrosis and early portalportal bridging fibrosis was observed in several patients. Nonetheless, the liver specimens of the other patients represented moderate macrovesicular steatosis without inflammatory infiltration [21].

Duarte-Neto et al. conducted the minimally-invasive ultrasound-guided autopsy of 10 patients with COVID-19. Coagulative necrosis in the central area and sinusoidal congestion with fibrin thrombi were observed in their histological examination of the liver [22].

Elsoukkary et al. documented histopathological abnormality in the hepatobiliary system in $61 \%$ of the patients with COVID-19. Mild lymphocytic inflammation was observed in $6(21 \%)$ cases. Large peculiar basophilic structures were detected in the sinusoids of $10(36 \%)$ cases, and intraductal trematode along with Fasciola hepatica was found in one (3\%) case [23].

In the study by Falasca et al., the microscopic examination of the liver tissue mainly revealed the extravasation of the red blood cells into the perisinusoidal space (or space of Disse) and sinusoidal congestion, and in a few 
Table 1. Characteristics of studies enrolled in this systematic review

\begin{tabular}{|c|c|c|c|c|c|c|c|c|c|c|}
\hline Authors & Countries & $\begin{array}{l}\text { Number } \\
\text { Deceased }\end{array}$ & Sex & Age (y) & $\begin{array}{l}\text { Average } \\
\text { Time } \\
\text { Between } \\
\text { Symptom } \\
\text { Onset to } \\
\text { Death } \\
\text { (d) }\end{array}$ & $\begin{array}{l}\text { Study } \\
\text { Design }\end{array}$ & $\begin{array}{c}\text { Average } \\
\text { Between } \\
\text { Death to } \\
\text { Autopsy } \\
\text { (h) }\end{array}$ & $\begin{array}{c}\text { Complete } \\
\text { Autopsy }\end{array}$ & $\begin{array}{l}\text { Mini- } \\
\text { mally in } \\
\text { Autopsy }\end{array}$ & $\begin{array}{c}\text { Number } \\
\text { of } \\
\text { Histology } \\
\text { Test }\end{array}$ \\
\hline $\begin{array}{l}\text { Sadegh } \\
\text { Beigee et } \\
\text { al. [18] }\end{array}$ & Iran & 27 & $\begin{array}{l}22 \text { male } \\
5 \text { female }\end{array}$ & $66(30-87)$ & 13 & $\begin{array}{l}\text { Case } \\
\text { series }\end{array}$ & $1-7$ & - & 27 & 27 \\
\hline $\begin{array}{l}\text { Beigmo- } \\
\text { hammadi } \\
\text { et al. [19] }\end{array}$ & Iran & 7 & $\begin{array}{c}5 \text { male } \\
2 \text { female }\end{array}$ & $68(46-84)$ & - & $\begin{array}{l}\text { Case } \\
\text { series }\end{array}$ & - & - & 7 & 7 \\
\hline $\begin{array}{c}\text { Bradley et } \\
\text { al. [20] }\end{array}$ & USA & 14 & $\begin{array}{c}\text { 6male } \\
\text { 8female }\end{array}$ & $73(42-84)$ & 7.92 & $\begin{array}{l}\text { Case } \\
\text { series }\end{array}$ & - & - & 14 & 14 \\
\hline $\begin{array}{c}\text { Buja et al. } \\
{[21]}\end{array}$ & USA & 23 & $\begin{array}{c}12 \text { male } \\
7 \text { female } \\
4 \text { not } \\
\text { specified }\end{array}$ & $34-76$ & - & $\begin{array}{l}\text { Case } \\
\text { series }\end{array}$ & $<24$ & - & 23 & 23 \\
\hline $\begin{array}{l}\text { Duarte- } \\
\text { Neto et } \\
\text { al. [22] }\end{array}$ & Brazil & 10 & $\begin{array}{c}5 \text { men } \\
5 \text { female }\end{array}$ & $69(33-83)$ & 10 & $\begin{array}{l}\text { Case } \\
\text { series }\end{array}$ & - & - & 10 & 10 \\
\hline $\begin{array}{c}\text { Elsouk- } \\
\text { kary et al. } \\
\text { [23] }\end{array}$ & USA & 32 & $\begin{array}{l}22 \text { male } \\
10 \text { female }\end{array}$ & $\begin{array}{c}68(30- \\
100)\end{array}$ & 21 & $\begin{array}{l}\text { Case } \\
\text { series }\end{array}$ & $43(5-382)$ & - & 32 & 32 \\
\hline $\begin{array}{c}\text { Falasca et } \\
\text { al. [24] }\end{array}$ & Italy & 18 & $\begin{array}{c}12 \text { male } \\
6 \text { female }\end{array}$ & $76(27-92)$ & - & $\begin{array}{l}\text { Case } \\
\text { series }\end{array}$ & - & & 22 & 22 \\
\hline $\begin{array}{c}\text { Rapkie- } \\
\text { wicz et al. } \\
\text { [25] }\end{array}$ & USA & 7 & $\begin{array}{c}3 \text { mle } \\
4 \text { female }\end{array}$ & $57(44-67)$ & 13 & $\begin{array}{l}\text { Case } \\
\text { series }\end{array}$ & $<24$ & - & 7 & 7 \\
\hline $\begin{array}{c}\text { Remme- } \\
\text { link et al. } \\
{[26]}\end{array}$ & Belgium & 17 & $\begin{array}{l}12 \text { males } \\
5 \text { female }\end{array}$ & $72(62-77)$ & 14 & $\begin{array}{l}\text { Case } \\
\text { series }\end{array}$ & $72-96$ & 17 & - & 17 \\
\hline $\begin{array}{l}\text { Schmit et } \\
\text { al. [27] }\end{array}$ & Belgium & 14 & $\begin{array}{l}10 \text { male } \\
4 \text { female }\end{array}$ & $63(30-83)$ & - & $\begin{array}{l}\text { Case } \\
\text { series }\end{array}$ & 20.2 & 14 & - & 14 \\
\hline $\begin{array}{l}\text { Sonzogni } \\
\text { et al. [28] }\end{array}$ & Italy & 48 & $\begin{array}{c}35 \text { male } \\
13 \text { female }\end{array}$ & $71(32-86)$ & - & $\begin{array}{l}\text { Case } \\
\text { series }\end{array}$ & 6 & 18 & 30 & 48 \\
\hline $\begin{array}{l}\text { Wich- } \\
\text { mann et } \\
\text { al. [29] }\end{array}$ & Germany & 12 & $\begin{array}{c}8 \text { male } \\
4 \text { female }\end{array}$ & $73(52-87)$ & - & $\begin{array}{c}\text { Prospec- } \\
\text { tive } \\
\text { cohort }\end{array}$ & $24-120$ & 12 & - & 12 \\
\hline $\begin{array}{c}\text { Zhao, } \\
\text { Chaohui } \\
\text { Lisa et al. } \\
\text { [30] }\end{array}$ & USA & 17 & $\begin{array}{l}10 \text { male } \\
7 \text { female }\end{array}$ & $65(44-85)$ & 21.75 & $\begin{array}{l}\text { Case } \\
\text { series }\end{array}$ & - & - & 17 & 17 \\
\hline $\begin{array}{l}\text { Schurink } \\
\text { et al. [31] }\end{array}$ & $\begin{array}{l}\text { Nether- } \\
\text { land }\end{array}$ & 21 & $\begin{array}{l}16 \text { male } \\
5 \text { female }\end{array}$ & $68(41-78)$ & 22 & $\begin{array}{c}\text { Prospec- } \\
\text { tive } \\
\text { cohort }\end{array}$ & $15(3-88)$ & 21 & - & 9 \\
\hline $\begin{array}{l}\text { Lagana et } \\
\text { al. [32] }\end{array}$ & Clombia & 40 & $\begin{array}{c}28 \text { male } \\
12 \text { female }\end{array}$ & $70(66-80)$ & - & $\begin{array}{l}\text { Case } \\
\text { series }\end{array}$ & - & 40 & - & 40 \\
\hline $\begin{array}{c}\text { Fassan et } \\
\text { al. [33] }\end{array}$ & Italy & 25 & $\begin{array}{c}14 \text { male } \\
11 \text { female }\end{array}$ & $82(61-97)$ & - & $\begin{array}{c}\text { Case } \\
\text { series }\end{array}$ & - & 25 & - & 50 \\
\hline
\end{tabular}


Table 2. Summary of the main findings of included articles on COVIDCOVID-19 related death

\begin{tabular}{|c|c|c|c|c|c|}
\hline Authors & Cause of Death & $\begin{array}{l}\text { Main Macroscopy } \\
\text { Findings in Liver }\end{array}$ & $\begin{array}{c}\text { Main } \\
\text { Microscopy Find- } \\
\text { ings in Liver }\end{array}$ & Other Findings & Comorbidities \\
\hline Sadegh Beigee et al. & COVID-19 & - & $\begin{array}{l}\text { Lymphoplasmacytic } \\
\text { inflammation and } \\
\text { interface hepatitis } \\
\text { fatty changes and } \\
\text { macrovesicular } \\
\text { steatosis }\end{array}$ & $\begin{array}{c}\text { Diffuse alveolar } \\
\text { damage and throm- } \\
\text { botic microangiopa- } \\
\text { thy of lung }\end{array}$ & $\begin{array}{l}\text { Hypertension fol- } \\
\text { lowed by diabetes } \\
\text { recently received } \\
\text { chemotherapy } \\
\text { for ovarian cancer } \\
\text { therapy }\end{array}$ \\
\hline $\begin{array}{l}\text { Beigmohammadi } \\
\text { et al. }\end{array}$ & COVID-19 & - & $\begin{array}{l}\text { Mild ballooning } \\
\text { degeneration of } \\
\text { hepatocytes and } \\
\text { congestion and } \\
\text { sinusoidal dilation, } \\
\text { macrovesicular } \\
\text { steatosis, and focal } \\
\text { confluent necrosis }\end{array}$ & $\begin{array}{c}\text { Diffuse alveolar } \\
\text { damage in lung, } \\
\text { Multinucleated cells } \\
\text { in alveolar spaces } \\
\text { and alveolar walls, } \\
\text { atypical enlarged } \\
\text { cells }\end{array}$ & $\begin{array}{l}\text { Hypertension, } \\
\text { rheumatoid arthritis } \\
\text { and bilateral periph- } \\
\text { eral ground-glass } \\
\text { opacities }\end{array}$ \\
\hline Bradley et al. & COVID-19 & - & $\begin{array}{l}\text { Mild periportal } \\
\text { interface hepatitis, } \\
\text { variable degrees of } \\
\text { acute congestion }\end{array}$ & $\begin{array}{l}\text { Diffuse alveolar } \\
\text { damage-type } \\
\text { lung injury in the } \\
\text { critical or organizing } \\
\text { phases }\end{array}$ & $\begin{array}{c}\text { Hypertension, } \\
\text { chronic kidney } \\
\text { disease, obstructive } \\
\text { sleep apnea, and } \\
\text { metabolic disease, } \\
\text { including diabetes } \\
\text { and obesity }\end{array}$ \\
\hline Buja et al. & COVID-19 & - & $\begin{array}{l}\text { Moderate macrove- } \\
\text { sicular steatosis, } \\
\text { lymphoplasmacytic } \\
\text { triaditis with portal } \\
\text { fibrosis, and early } \\
\text { portal-portal bridg- } \\
\text { ing fibrosis }\end{array}$ & $\begin{array}{l}\text { Major pulmonary } \\
\text { thromboembolic } \\
\text { with pulmonary } \\
\text { infarcts and hemor- } \\
\text { rhage }\end{array}$ & $\begin{array}{c}\text { Hypertension, } \\
\text { obesity, Type II } \\
\text { diabetes mellitus }\end{array}$ \\
\hline Duarte-Neto et al. & COVID-19 & - & $\begin{array}{l}\text { Steatosis of hepa- } \\
\text { tocytes, portal tract } \\
\text { inflammatory infil- } \\
\text { trate, Centrilobular } \\
\text { congestion, focal } \\
\text { necrosis, Kupffer } \\
\text { cell hypertrophy, } \\
\text { haemophagocyto- } \\
\text { sis, and Sinusoids } \\
\text { with neutrophilic } \\
\text { infiltrate }\end{array}$ & $\begin{array}{l}\text { Exudative/prolifera- } \\
\text { tive Diffuse Alveolar } \\
\text { Damage (DAD) }\end{array}$ & $\begin{array}{l}\text { Hypertension, dia- } \\
\text { betes mellitus, renal } \\
\text { arteriolosclerosis, } \\
\text { cardiomyocytes } \\
\text { hypertrophy, myo- } \\
\text { cardial fibrosis, focal } \\
\text { glomerular sclero- } \\
\text { sis, liver steatosis, } \\
\text { and cerebral small } \\
\text { vessel disease }\end{array}$ \\
\hline Elsoukkary et al. & COVID-19 & & $\begin{array}{l}\text { Large basophilic } \\
\text { structures in sinu- } \\
\text { soidal endothe- } \\
\text { lium and increased } \\
\text { immunoblast-like } \\
\text { cells in lymph nodes }\end{array}$ & $\begin{array}{l}\text { Alveolar neutrophils } \\
\text { and evidence of } \\
\text { organizing pneu- } \\
\text { monia. All cases } \\
\text { had some degree of } \\
\text { type II pneumocyte } \\
\text { hyperplasia with } \\
\text { reactive atypia and } \\
\text { bronchial squamous } \\
\text { metaplasia. }\end{array}$ & $\begin{array}{l}\text { Hypertension, } \\
\text { hyperlipidemia, } \\
\text { diabetes, obe- } \\
\text { sity, Coronary } \\
\text { artery disease, CHF, } \\
\text { chronic obstructive } \\
\text { pulmonary disease, } \\
\text { asthma, obstructive } \\
\text { pulmonary apnea, } \\
\text { stroke, dementia, } \\
\text { cancer }\end{array}$ \\
\hline Falasca et al. & COVID-19 & $\begin{array}{l}\text { Parenchymal con- } \\
\text { gestion }\end{array}$ & $\begin{array}{l}\text { Inflammatory } \\
\text { infiltrate, } \\
\text { Steatosis }\end{array}$ & $\begin{array}{l}\text { Pulmonary and } \\
\text { cardiovascular in- } \\
\text { volvement is domi- } \\
\text { nant pathological } \\
\text { features }\end{array}$ & $\begin{array}{l}\text { Chronic Obstruc- } \\
\text { tive Pulmonary } \\
\text { disease Cardiac } \\
\text { disease Malignancy } \\
\text { Hypertension Dia- } \\
\text { betes Schizophrenia } \\
\text { Kidney disease }\end{array}$ \\
\hline Rapkiewicz et al. & COVID-19 & - & $\begin{array}{l}\text { Mild, macrove- } \\
\text { sicular steatosis, } \\
\text { numerous platelet- } \\
\text { fibrin microthrombi } \\
\text { in hepatic sinusoids, } \\
\text { Cirrhosis }\end{array}$ & $\begin{array}{l}\text { Platelet-rich } \\
\text { thrombi in the } \\
\text { pulmonary, hepatic, } \\
\text { renal, and cardiac } \\
\text { microvasculature }\end{array}$ & $\begin{array}{l}\text { History of cardiac } \\
\text { arrest } \\
\text { Hypertension } \\
\text { High cholesterol } \\
\text { Diabetes, Obesity, } \\
\text { Use of Anticoagu- } \\
\text { lant }\end{array}$ \\
\hline
\end{tabular}


Table 2. (continued)

\begin{tabular}{|c|c|c|c|c|c|}
\hline Authors & $\begin{array}{l}\text { Cause of } \\
\text { Death }\end{array}$ & $\begin{array}{l}\text { Main Macros- } \\
\text { copy Findings } \\
\text { in Liver }\end{array}$ & $\begin{array}{l}\text { Main } \\
\text { Microscopy Findings } \\
\text { in Liver }\end{array}$ & Other Findings & Comorbidities \\
\hline $\begin{array}{l}\text { Remmelink } \\
\text { et al. }\end{array}$ & COVID-19 & Hepatomegaly & $\begin{array}{c}\text { Congestion hepatopa- } \\
\text { thy, } \\
\text { Cirrhosis and hepatic } \\
\text { steatosis, }\end{array}$ & $\begin{array}{c}\text { Eearly-stage diffuse alveolar } \\
\text { damage (DAD), microthrom- } \\
\text { bi in small lung arteries. } \\
\text { Late-stage DAD, atypical } \\
\text { pneumocytes, and acute } \\
\text { pneumonia myocardial } \\
\text { infarctions }\end{array}$ & $\begin{array}{l}\text { Hypertension, diabe- } \\
\text { tes, cerebrovascular } \\
\text { disease, coronary } \\
\text { artery disease, and } \\
\text { solid cancer }\end{array}$ \\
\hline Schmit et al. & COVID-19 & $\begin{array}{l}\text { Pale and yel- } \\
\text { lowish and a } \\
\text { nutmeg ap- } \\
\text { pearance }\end{array}$ & $\begin{array}{l}\text { Centro lobular necrosis, } \\
\text { discreet to moder- } \\
\text { ate lobular or portal } \\
\text { inflammation. Steatosis } \\
\text { Cholestasis and discrete } \\
\text { bile duct proliferation }\end{array}$ & - & $\begin{array}{l}\text { Hypertension and } \\
\text { heart disease), high } \\
\text { BMI, or obesity. }\end{array}$ \\
\hline Sonzogni et al. & COVID-19 & $\begin{array}{l}\text { Minimal inflam- } \\
\text { mation }\end{array}$ & $\begin{array}{l}\text { Portal vein parietal } \\
\text { fibrosis Herniated } \\
\text { portal vein in periportal } \\
\text { parenchyma, Peripor- } \\
\text { tal abnormal vessels, } \\
\text { Fibrosis, Lobular, and } \\
\text { portal inflammation }\end{array}$ & $\begin{array}{l}\text { Lung parenchyma, cardio- } \\
\text { vascular system, coagulation } \\
\text { cascade, and the endothelial } \\
\text { layer of blood vessels }\end{array}$ & $\begin{array}{l}\text { Hypertension, Car- } \\
\text { diovascular Disease } \\
\text { (different than hyper- } \\
\text { tension), Diabetes, } \\
\text { Obesity, Kidney and } \\
\text { pulmonary disease }\end{array}$ \\
\hline $\begin{array}{l}\text { Wichmann } \\
\text { et al. }\end{array}$ & COVID-19 & $\begin{array}{l}\text { Hepatomegaly, } \\
\text { shock liver, } \\
\text { chronic conges- } \\
\text { tion, fatty } \\
\text { changes }\end{array}$ & $\begin{array}{l}\text { Viral RNA at high titers } \\
\text { in other organs in many } \\
\text { patients }\end{array}$ & $\begin{array}{l}\text { Diffuse alveolar damage in } \\
\text { lung }\end{array}$ & $\begin{array}{l}\text { Obesity, coronary } \\
\text { heart disease, } \\
\text { asthma or chronic } \\
\text { obstructive pul- } \\
\text { monary disease, } \\
\text { peripheral artery } \\
\text { disease, diabetes, } \\
\text { neurodegenerative } \\
\text { diseases }\end{array}$ \\
\hline Zhao et al. & COVID-19 & - & $\begin{array}{l}\text { Platelet-fibrin thrombi } \\
\text { in sinusoid, central } \\
\text { vein or portal vein, } \\
\text { Rare megakaryocytes } \\
\text { in sinusoids, Histiocytic } \\
\text { hyperplasia, Steatosis, } \\
\text { Lobular inflammation, } \\
\text { mild Portal tract inflam- } \\
\text { mation, Ischemic-type } \\
\text { hepatic necrosis, }\end{array}$ & $\begin{array}{l}\text { Abnormal liver-associated } \\
\text { enzymes were observed in } \\
\text { 12/17 cases of COVID-19 } \\
\text { infection. }\end{array}$ & $\begin{array}{c}\text { Coronary artery } \\
\text { disease; congestive } \\
\text { heart failure; chronic } \\
\text { kidney failure; type II } \\
\text { diabetes; hyperlipid- } \\
\text { emia; hypertension; } \\
\text { deep vein thrombosis }\end{array}$ \\
\hline Schurink et al. & COVID-19 & & $\begin{array}{l}\text { Stenosis, Minor portal } \\
\text { and lobular inflamma- } \\
\text { tion, confluent necrosis, } \\
\text { periportal and per } \\
\text { sinusoidal fibrosis, and } \\
\text { true bridging }\end{array}$ & $\begin{array}{l}\text { Extensive inflammatory } \\
\text { response, also thrombi and } \\
\text { neutrophilic plugs were } \\
\text { present in the lungs, heart, } \\
\text { kidneys, liver, spleen, and } \\
\text { brain and were most fre- } \\
\text { quently observed late in the } \\
\text { disease course }\end{array}$ & $\begin{array}{l}\text { Diabetes, Cardio- } \\
\text { vascular disease, } \\
\text { Chronic obstructive } \\
\text { pulmonary disease, } \\
\text { Asthma, Active solid } \\
\text { malignancy, Active } \\
\text { hematological malig- } \\
\text { nancy, and others. }\end{array}$ \\
\hline Lagana et al. & COVID-19 & $\begin{array}{l}\text { Fibrosis, } \\
\text { abscesses, } \\
\text { the remaining } \\
\text { livers showed } \\
\text { varying degrees } \\
\text { of steatosis, } \\
\text { congestion, and } \\
\text { ischemia }\end{array}$ & $\begin{array}{l}\text { Hepatic steatosis, mild } \\
\text { acute hepatitis, and } \\
\text { portal inflammation }\end{array}$ & $\begin{array}{l}\text { Acute lung injury, Lobular } \\
\text { necroinflammation includes } \\
\text { diffuse alveolar damage, } \\
\text { including exudative and pro- } \\
\text { liferative phases and acute } \\
\text { fibrinous and organizing } \\
\text { pneumonia. }\end{array}$ & $\begin{array}{c}\text { Chronic liver disease, } \\
\text { Diabetes, Hyperten- } \\
\text { sion, Chronic kidney } \\
\text { disease, and Cardiac } \\
\text { disease }\end{array}$ \\
\hline Fassan et al. & COVID-19 & $\begin{array}{l}\text { Macro-vesicular } \\
\text { Centro acinar } \\
\text { steatosis }\end{array}$ & $\begin{array}{l}\text { Parenchymal sinusoids, } \\
\text { hepatic steatosis, mild/ } \\
\text { absent lymphocytic } \\
\text { hepatitis, sinusoidal } \\
\text { ectasia }\end{array}$ & - & $\begin{array}{l}\text { Obesity, neoplastic } \\
\text { disease, bedridden, } \\
\text { hypertension }\end{array}$ \\
\hline
\end{tabular}


cases, the congestion of the small veins and hepatocyte necrosis; infiltration were also detected [24].

Mild macrovesicular steatosis without significant inflammation or ballooning that showed steatohepatitis was reported in Rapkiewicz et al. Platelet- fibrin microthrombi in hepatic sinusoids, a common finding in $86 \%$ of the deceased patients in this review study. The aggregation of platelets was observed in the portal veins with a hepatic vein thrombosis in $14 \%$ of the patients [25].

In the study by Remmelink et al. on 17 deceased patients with the complete autopsy, the postmortem microscopic examination of the liver samples revealed congestive hepatopathy and steatosis; however, no patchy necrosis, hepatitis, or lobular lymphocytic infiltration was reported [26].

Centrilobular necrosis associated with discrete mild to moderate lobular and portal inflammation of the liver tissue was observed in $12(86 \%)$ of the deceased patients with COVID-19 examined by Schmit et al. Moreover, discrete bile duct proliferation and cholestasis were reported in 5(36\%) cases [27].

Sonzogni et al. described the postmortem liver findings of 48 patients with COVID-19. Vascular thrombosis with lobular architecture was found in all the samples. Additionally, 29(60\%) patients had partial portal vein fibrosis (phlebosclerosis). The herniated portal vein in the periportal parenchyma defected in $36(75 \%)$ of the patients. The other findings from the microscopic evaluation of the liver tissues included fibrosis, varying degrees of lobular and portal inflammation, and parenchymal confluent necrosis, i.e., manifested during 18 complete autopsies and 30 minimally-invasive autopsies [28].

Schurink et al. performed a microscopic investigation of the liver in 21 deceased patients. Accordingly, they suggested neutrophilic plugs, i.e., composed of the aggregates of Neutrophil Extracellular Traps (NETs) with or without platelets. These aggregates were observed in several organs during the complete autopsies, such as the liver. Minor portal and labor inflammation were reported in $62 \%$ and $29 \%$ of the cadavers, respectively. Periportal and perisinusoidal fibrosis were detected in $38 \%$ of the patients; true bridging fibrosis was observed in $14 \%$, probably due to pre-existing liver injuries [31].

In the study by Lagana et al., vascular pathology, including sinusoidal microthrombi, was observed in $15 \%$ of the patients who expired due to COVID-19. The authors concluded that the deceased patients had biochemi- cal evidence of hepatitis, macrovesicular steatosis, mild acute hepatitis, and mild portal inflammation. They also detected viral RNA in their liver samples [32].

Fassan et al. revealed that the main histopathological findings in cadavers with COVID-19 infection were mild or absent lymphocytic hepatitis, the activation of (pigmented) Kupffer cells, hepatocellular regenerative changes, and steatosis in autopsy specimens [33].

\section{Discussion}

This systematic review represents macroscopic and microscopic findings in the liver of deceased patients with COVID-19 in the postmortem examinations and reports their comorbidities. The main manifestations of the liver were moderate macro and microvesicular steatosis with mild sinusoidal dilation, active lobular and portal vein thrombosis, mildly-increased lymphocyte infiltration in the sinusoidal space, and multifocal hepatic necrosis. Any pre-existing chronic liver disease and comorbidities exacerbate COVID-19 signs of hyperinflammatory reactions and may contribute to hepatic damage.

Since postmortem examinations were avoided in numerous countries in the initial phase of the COVID-19 pandemic, there was a dire need for information obtained from autopsy to improve the management and therapy strategy of mild to severely infected patients $[34,35]$. As of the date of writing this manuscript, there is no exact postmortem examination protocol for COVID-19.

To date, pathological investigations of COVID-19 have mainly focused on pulmonary findings in death due to COVID-19, and histopathological reports on the other organs discussed in the literature are also essential.

Studies indicated that $2 \%-11 \%$ of COVID-19 patients had comorbid chronic liver diseases, and 37.2\%-76.3\% of them have had impaired liver function [36-38].

In this study, steatosis was the most common histopathological finding, which can be explained by concomitant diabetes, hyperlipidemia, and obesity. These conditions are the most significant risk factors for developing fatty liver that causes simple steatosis, advanced fibrosis, cryptogenic Cirrhosis, and hepatocellular carcinoma. Nonetheless, they demonstrate a pattern specific to hepatic damage by COVID-19 that is consistent with the results of other studies $[39,40]$.

Liver injury in mild cases of COVID-19 is often temporary and can return to normal even without special 
treatment; however, protective drugs are necessary for patients with severe liver damage [41, 42].

In this investigation, the gross findings of the liver of the cadavers included hepatomegaly, portal fibrosis, liver necrosis, and central vein thrombosis, i.e., similar to the results of other studies [43, 44].

According to our systematic review, the most common comorbidities were hypertension, metabolic diseases, including diabetes and obesity, and cardiovascular and respiratory diseases, i.e., consistent with the results of other studies $[45,46]$. The mortality rate was higher in patients with hypertension, diabetes, a history of cardiovascular diseases, and chronic diseases (e.g., renal \& cerebrovascular diseases), consistent with other studies' results $[45,47]$.

Just like other systematic reviews, this study has several limitations. First, due to the increased number of publications daily, missing published articles was an important challenge in this research. Secondly, clinical data, such as laboratory findings, were not reported in the reviewed articles for all the patients. Thirdly, the postmortem examination results describing the patients with the severe disease might not be found in surviving patients. Finally, the pathological findings of the liver in patients deceased with COVID-19 were represented with different terminologies and various levels of detail, and there was no uniformity in the procedures of autopsy in the studies. Therefore, data comparison is faced with a potential limitation. There are no postmortem diagnostic protocols available based on population-based studies, and health care management processes need to be standardized.

However, we described the macroscopic and histologic characteristics and complications of COVID-19 in the liver of deceased patients. Further investigations are required to evaluate better the possible factors that contribute to liver injury in these patients and understand the association between hepatic findings, drug administration, comorbidities, and COVID-19 based on clinical and histological observations. Therefore, several studies with long-term follow-ups are required to describe the extent and cause of death in patients with liver damage due to COVID-19. The effects of COVID-19 in chronic liver disease need detailed investigations, especially in postmortem examinations of the cadavers.

\section{Conclusion}

Hepatic manifestations can occur in COVID-19 infection with varied macroscopic and microscopic findings, including hepatomegaly, hepatic steatosis, lobular cholestasis, portal fibrosis, lymphocytic infiltrate, and ductular lesions proliferation, and acute liver cell necrosis with vein thrombosis. Additionally, patients with other comorbidities and abnormal liver function have a high risk of developing severe complications and mortality when infected. Nonetheless, the exact mechanisms of liver injury with COVID-19 remain unclear. The liver function must be monitored continuously during illness to avoid severe complications and better manage the patients during this pandemic.

\section{Ethical Considerations}

\section{Compliance with ethical guidelines}

The Ethics Committee approved the study of Legal Medicine Organization, Tehran, Iran (Code: IR.LMO. REC.1400. 006)

\section{Funding}

This research received no specific grant from funding agencies in the public, commercial, or non-profit sectors.

\section{Author's contributions}

All authors contributed equally to this work.

\section{Conflict of interest}

The authors reported no conflicts of interest.

\section{Acknowledgements}

The authors gratefully acknowledge Tehran University of Medical Sciences for their guidance. Special thanks to Amir Hossein Behnoush, for his perseverance in revision of the manuscript.

\section{References}

[1] Chen N, Zhou M, Dong X, Qu J, Gong F, Han Y, et al. Epidemiological and clinical characteristics of 99 cases of 2019 novel coronavirus pneumonia in Wuhan, China: A descriptive study. Lancet. 2020; 395(10223):507-13. [DOI:10.1016/ S0140-6736(20)30211-7]

[2] World Health Organization (WHO). Listings of WHO's response to COVID-19; Accessed 21 Dec 2020. https://www. who.int/news/item/29-06-2020-covid timeline

[3] Sohrabi C, Alsafi Z, O'Neill N, Khan M, Kerwan A, Al-Jabir A, et al. World Health Organization declares global emergency: 
A review of the 2019 novel coronavirus (COVID-19). Int J Surg 2020; 76:71-6. [DOI:10.1016/j.ijsu.2020.02.034] [PMID] [PMCID]

[4] BesharatS, Malekpour Alamda N, Dadashzadeh N, Talaie R, MousaviSS, Barzegar A, et al. Clinical and demographic characteristics of patients with COVID-19 who died in Modarres Hospital. Maced J Med Sci. 2020; 8(T1):144-9. [DOI:10.3889/ oamjms.2020.5013]

[5] Keten D, Okdemir E, Keten A. Precautions in postmortem examinations in Covid-19 - Related deaths: Recommendations from Germany. J Forensic Leg Med. 2020; 73:102000. [DOI:10.1016/j.jflm.2020.102000] [PMID] [PMCID]

[6] Docherty AB, Harrison EM, Green CA, Hardwick HE, Pius R, Norman L, et al. Features of 20133 UK patients in hospital with covid-19 using the ISARIC WHO Clinical Characterisation Protocol: Prospective observational cohort study. BM] (Clinical research ed). 2020; 369:m1985. [DOI:10.1136/bmj. m1985] [PMID] [PMCID]

[7] Wu C, Chen X, Cai Y, Xia J, Zhou X, Xu S, et al. Risk factors associated with acute respiratory distress syndrome and death in patients with coronavirus disease 2019 pneumonia in Wuhan, China. JAMA Intern Med. 2020; 180(7):934-43. [DOI:10.1001/jamainternmed.2020.0994] [PMID] [PMCID]

[8] Cummings MJ, Baldwin MR, Abrams D, Jacobson SD, Meyer BJ, Balough EM, et al. Epidemiology, clinical course, and outcomes of critically ill adults with COVID-19 in New York City: A prospective cohort study. Lancet. 2020; 395(10239):1763-70. [DOI:10.1016/S0140-6736(20)31189-2]

[9] Daneshvar M, Dadashzadeh N, Ahmadpour M, Ragati Haghi H, Rahmani V, Frouzesh M, et al. Lessons of mortality following COVID-19 epidemic in the United States especially in the geriatrics. J Nephropharmacol. 2021; 10(1):e06. [DOI:10.34172/npj.2021.06]

[10] Xu L, Liu J, Lu M, Yang D, Zheng X. Liver injury during highly pathogenic human coronavirus infections. Liver Int. 2020; 40(5):998-1004. [DOI:10.1111/liv.14435] [PMID] [PMCID]

[11] Zhang Y, Zheng L, Liu L, Zhao M, Xiao J, Zhao Q. Liver impairment in COVID-19 patients: A retrospective analysis of 115 cases from a single centre in Wuhan city, China. Liver Int. 2020; 40(9):2095-103. [DOI:10.1111/liv.14455] [PMID]

[12] Li Y, Xiao SY. Hepatic involvement in COVID-19 patients: Pathology, pathogenesis, and clinical implications. J Med Virol. 2020; 92(9):1491-4. [DOI:10.1002/jmv.25973] [PMID]

[13] Shi H, Han X, Jiang N, Cao Y, Alwalid O, Gu J, et al. Radiological findings from 81 patients with COVID-19 pneumonia in Wuhan, China: A descriptive study. Lancet Infect Dis. 2020; 20(4):425-34. [DOI:10.1016/S1473-3099(20)30086-4] [PMID]

[14] Bangash MN, Patel J, Parekh D. COVID-19 and the liver: Little cause for concern. Lancet Gastroenterol Hepatol. 2020; 5(6):529-30. [DOI:10.1016/S2468-1253(20)30084-4] [PMID]

[15] Chai X, Hu L, Zhang Y, Han W, Lu Z, Ke A, et al. Specific ACE2 expression in cholangiocytes may cause liver damage after 2019-nCoV infection. bioRxiv. 2020. [DOI:10.1101/2020.02.03.931766]

[16] Liberati A, Altman DG, Tetzlaff J, Mulrow C, Gøtzsche PC, Ioannidis JP, et al. The PRISMA statement for reporting systematic reviews and meta-analyses of studies that evaluate healthcare interventions: Explanation and elaboration. BMJ. 2009; 339:b2700. [DOI:10.1136/bmj.b2700] [PMID] [PMCID]
[17] Murad MH, Sultan S, Haffar S, Bazerbachi F. Methodological quality and synthesis of case series and case reports. BMJ Evid Based Med. 2018; 23(2):60-3. [DOI:10.1136/bmjebm-2017-110853] [PMID] [PMCID]

[18] Sadegh Beigee F, Pourabdollah Toutkaboni M, Khalili N, Nadji SA, Dorudinia A, Rezaei M, et al. Diffuse alveolar damage and thrombotic microangiopathy are the main histopathological findings in lung tissue biopsy samples of COVID-19 patients. Pathol Res Pract. 2020; 216(10):153228. [DOI:10.1016/j.prp.2020.153228] [PMID] [PMCID]

[19] Beigmohammadi MT, Jahanbin B, Safaei M, Amoozadeh L, Khoshavi M, Mehrtash V, et al. Pathological findings of postmortem biopsies from lung, heart, and liver of 7 deceased COVID-19 patients. Int J Surg Pathol. 2021; 29(2):135-45. [DOI:10.1177/1066896920935195] [PMID] [PMCID]

[20] Bradley BT, Maioli H, Johnston R, Chaudhry I, Fink SL, $\mathrm{Xu} \mathrm{H}$, et al. Histopathology and ultrastructural findings of fatal COVID-19 infections in Washington State: A case series. Lancet. 2020; 396(10247):320-32. [DOI:10.1016/S01406736(20)31305-2] [PMID]

[21] Buja LM, Wolf DA, Zhao B, Akkanti B, McDonald M, Lelenwa $\mathrm{L}$, et al. The emerging spectrum of cardiopulmonary pathology of the coronavirus disease 2019 (COVID-19): Report of 3 autopsies from Houston, Texas, and review of autopsy findings from other United States cities. Cardiovasc Pathol. 2020; 48:107233. [DOI:10.1016/j.carpath.2020.107233] [PMID] [PMCID]

[22] Duarte-Neto AN, Monteiro RAA, da Silva LFF, Malheiros DMAC, de Oliveira EP, Theodoro-Filho J, et al. Pulmonary and systemic involvement in COVID-19 patients assessed with ultrasound-guided minimally invasive autopsy. Histopathology. 2020; 77(2):186-97. [DOI:10.1111/his.14160] [PMID] [PMCID]

[23] Elsoukkary SS, Mostyka M, Dillard A, Berman DR, Ma LX, Chadburn A, et al. Autopsy findings in 32 patients with COVID-19: A single-institution experience. Pathobiology. 2021; 88(1):56-68. [DOI:10.1159/000511325] [PMID] [PMCID]

[24] Falasca L, Nardacci R, Colombo D, Lalle E, Di Caro A Nicastri E, et al. Postmortem findings in Italian patients with COVID-19: A descriptive full autopsy study of cases with and without comorbidities. J Infect Dis. 2020; 222(11):1807-15. [DOI:10.1093/infdis/jiaa578] [PMID] [PMCID]

[25] Rapkiewicz AV, Mai X, Carsons SE, Pittaluga S, Kleiner $\mathrm{DE}$, Berger JS, et al. Megakaryocytes and platelet-fibrin thrombi characterize multi-organ thrombosis at autopsy in COVID-19: A case series. EClinicalMedicine. 2020; 24:100434. [DOI:10.1016/j.eclinm.2020.100434] [PMID] [PMCID]

[26] Remmelink M, De Mendonça R, D'Haene N, De Clercq S, Verocq C, Lebrun L, et al. Unspecific post-mortem findings despite multiorgan viral spread in COVID-19 patients. Crit Care. 2020; 24(1):495. [DOI:10.1186/s13054-020-03218-5] [PMID] [PMCID]

[27] Schmit G, Lelotte J, Vanhaebost J, Horsmans Y, Van Bockstal M, Baldin P. The liver in COVID-19-related death: Protagonist or innocent bystander? Pathobiology. 2021; 88(1):88-94. [DOI:10.1159/000512008] [PMID] [PMCID]

[28] Sonzogni A, Previtali G, Seghezzi M, Grazia Alessio M, Gianatti A, Licini L, et al. Liver histopathology in severe COVID 19 respiratory failure is suggestive of vascular alterations. Liver Int 2020; 40(9):2110-6. [DOI:10.1111/liv.14601] [PMID] [PMCID] 
[29] Wichmann D, Sperhake JP, Lütgehetmann M, Steurer S, Edler C, Heinemann A, et al. Autopsy findings and venous thromboembolism in patients with COVID-19: A prospective cohort study. Ann Intern Med. 2020; 173(4):268-77. [DOI:10.7326/M20-2003] [PMID] [PMCID]

[30] Zhao CL, Rapkiewicz A, Maghsoodi-Deerwester M, Gupta M, Cao W, Palaia T, et al. Pathological findings in the postmortem liver of patients with coronavirus disease 2019 (COVID-19). Hum Pathol. 2021; 109:59-68. [DOI:10.1016/j. humpath.2020.11.015] [PMID] [PMCID]

[31] Schurink B, Roos E, Radonic T, Barbe E, Bouman CSC, de Boer $\mathrm{HH}$, et al. Viral presence and immunopathology in patients with lethal COVID-19: A prospective autopsy cohort study. Lancet Microbe. 2020; 1(7):e290-9. [DOI:10.1016/S26665247(20)30144-0]

[32] Lagana SM, Kudose S, Iuga AC, Lee MJ, Fazlollahi L, Remotti HE, et al. Hepatic pathology in patients dying of COVID-19: A series of 40 cases including clinical, histologic, and virologic data. Mod Pathol. 2020; 33(11):2147-55. [DOI:10.1038/ s41379-020-00649-x] [PMID] [PMCID]

[33] Fassan M, Mescoli C, Sbaraglia M, Guzzardo V, Russo FP, Fabris R, et al. Liver histopathology in COVID-19 patients: A mono-Institutional series of liver biopsies and autopsy specimens. Pathol Res Pract. 2021; 221:153451. [DOI:10.1016/j. prp.2021.153451] [PMID] [PMCID]

[34] Tombolini A, Scendoni R. SARS-CoV-2-related deaths in routine forensic autopsy practice: Histopathological patterns. Int J Legal Med. 2020; 134(6):2205-8. [DOI:10.1007/s00414020-02354-5] [PMID] [PMCID]

[35] Ledford H. Autopsy slowdown hinders quest to determine how coronavirus kills. Nature. 2020. [DOI:10.1038/d41586020-01355-z] [PMID]

[36] Zhang C, Shi L, Wang FS. Liver injury in COVID-19: Management and challenges. Lancet Gastroenterol Hepatol. 2020; 5(5):428-30. [DOI:10.1016/S2468-1253(20)30057-1]

[37] Fan Z, Chen L, Li J, Cheng X, Yang J, Tian C, et al. Clinical features of COVID-19-related liver functional abnormality. Clin Gastroenterol Hepatol. 2020; 18(7):1561-6. [DOI:10.1016/j.cgh.2020.04.002] [PMID] [PMCID]

[38] Cai Q, Huang D, Yu H, Zhu Z, Xia Z, Su Y, et al. COVID-19: Abnormal liver function tests. J Hepatol. 2020; 73(3):566-74. [DOI: 10.1016/j.jhep.2020.04.006] [PMID] [PMCID]

[39] Martines RB, Ritter JM, Matkovic E, Gary J, Bollweg $\mathrm{BC}$, Bullock $\mathrm{H}$, et al. Pathology and pathogenesis of SARSCoV-2 associated with fatal coronavirus disease, United States. Emerg Infect Dis. 2020; 26(9):2005-15. [DOI:10.3201/ eid2609.202095] [PMID] [PMCID]

[40] Milroy CM. Fatty liver and the forensic pathologist. Acad Forensic Pathol. 2018; 8(2):296-310. [DOI:10.1177/1925362118782061] [PMID] [PMCID]

[41] D'Amico F, Baumgart DC, Danese S, Peyrin-Biroulet L. Diarrhea during COVID-19 infection: Pathogenesis, epidemiology, prevention, and management. Clin Gastroenterol Hepatol. 2020; 18(8):1663-72. [DOI:10.1016/j.cgh.2020.04.001] [PMID] [PMCID]

[42] Nobel YR, Phipps M, Zucker J, Lebwohl B, Wang TC, Sobieszczyk ME, et al. Gastrointestinal symptoms and coronavirus disease 2019: A case-control study from the United States.
Gastroenterology. 2020; 159(1):373-5.e2. [DOI:10.1053/j.gastro.2020.04.017] [PMID] [PMCID]

[43] Eketunde AO, Mellacheruvu SP, Oreoluwa P. a review of postmortem findings in patients with COVID-19. Cureus. 2020; 12(7):e9438. [DOI:10.7759/cureus.9438]

[44] Lax SF, Skok K, Zechner P, Kessler HH, Kaufmann N, Koelblinger C, et al. Pulmonary arterial thrombosis in COVID-19 with fatal outcome: Results from a prospective, singlecenter, clinicopathologic case series. Ann Intern Med. 2020; 173(5):350-61. [DOI:10.7326/M20-2566] [PMID] [PMCID]

[45] Jothimani D, Venugopal R, Abedin MF, Kaliamoorthy I, Rela M. COVID-19 and the liver. J Hepatol. 2020; 73(5):1231-40. [DOI:10.1016/j.jhep.2020.06.006] [PMID] [PMCID]

[46] Yang J, Zheng Y, Gou X, Pu K, Chen Z, Guo Q, Ji R, et al. Prevalence of comorbidities and its effects in patients infected with SARS-CoV-2: A systematic review and meta-analysis. Int J Infect Dis. 2020; 94:91-5. [DOI:10.1016/j.iiid.2020.03.017] [PMID] [PMCID]

[47] Chen T, Wu D, Chen H, Yan W, Yang D, Chen G, et al. Clinical characteristics of 113 deceased patients with coronavirus disease 2019: Retrospective study. BMJ. 2020; 368:m1091. [DOI:10.1136/bmj.m1091] [PMID] [PMCID] 
This Page Intentionally Left Blank 